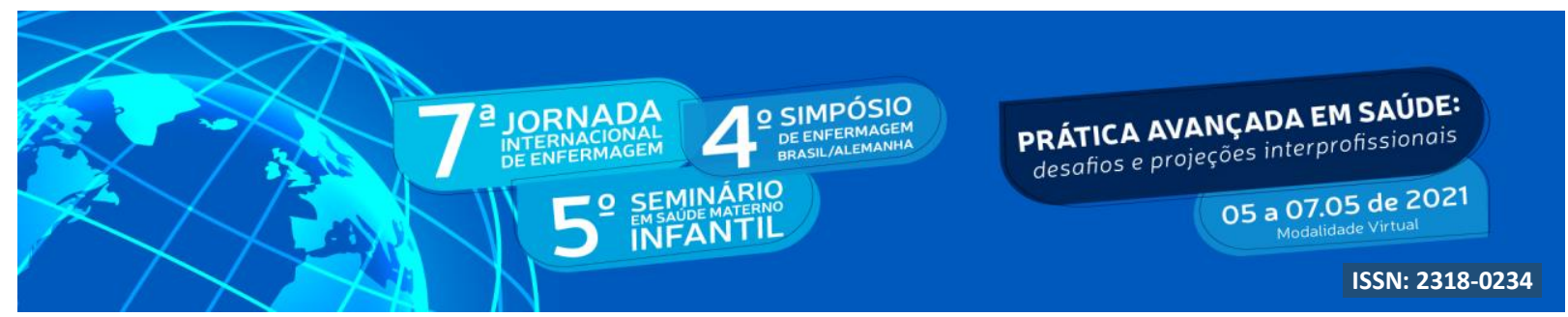

DOI: http://doi.org/10.48195/jie2021-066

\title{
APLICATIVO DE PRIMEIROS SOCORROS PARA INJÚRIAS NÃO INTENCIONAIS PEDIÁTRICAS ${ }^{1}$
}

\author{
Márian Oleques Pires²; Amanda Weissheimer³; Dirce Stein Backes ${ }^{4}$
}

\begin{abstract}
RESUMO
Os smartphones tornaram-se uma possibilidade crescente de propagação de informações de boas práticas em saúde. Objetiva-se, assim, descrever o processo de desenvolvimento de um aplicativo de primeiros socorros para injúrias não intencionais pediátricas. Trata-se de um estudo de produção tecnológica, do tipo prototipagem, desenvolvido no Sul do Brasil, entre os anos de 2018 e 2019, a partir de quatro etapas: Construção e validação do protocolo de primeiros socorros pediátricos; modelagem do protótipo; validação da prototipagem; implementação do aplicativo. Com base na técnica Delphi, o aplicativo foi considerado por especialistas pertinente em relação às dimensões funcionalidade, confiabilidade, acessibilidade, factibilidade e inovação. O dispositivo móvel de primeiros socorros para injúrias não intencionais pediátricas, servirá de apoio em situações de emergência pediátrica tanto para os cuidadores familiares, quanto para os profissionais da área da saúde e da educação.
\end{abstract}

Palavras-chave: Primeiros socorros; Cuidados de Enfermagem; Aplicativos Móveis; Tecnologia da Informação; Inovação tecnológica.

\section{ABSTRACT}

Taking advantage of the continuous use of smartphones has become a possibility of reproducing information on good health practices, this work aims to describe the process of developing a first aid application for pediatric unintentional injuries. It is a study of technological production, of the prototyping type, developed in the south of Brazil between the years 2018 and 2019, based on four

\footnotetext{
${ }^{1}$ Trabalho de produção tecnológica

${ }^{2}$ Mestranda em Saúde Materno Infantil. Universidade Franciscana. E-mail:piresoleques@ gmail.com

${ }^{3}$ Mestre em Saúde Materno Infantil. Universidade Franciscana. E-mail: amandiinhaweiss@gmail.com

${ }^{4}$ Orientador. Doutora em Enfermagem. Universidade Franciscana. E-mail: backesdirce@ufn.edu.br
} 


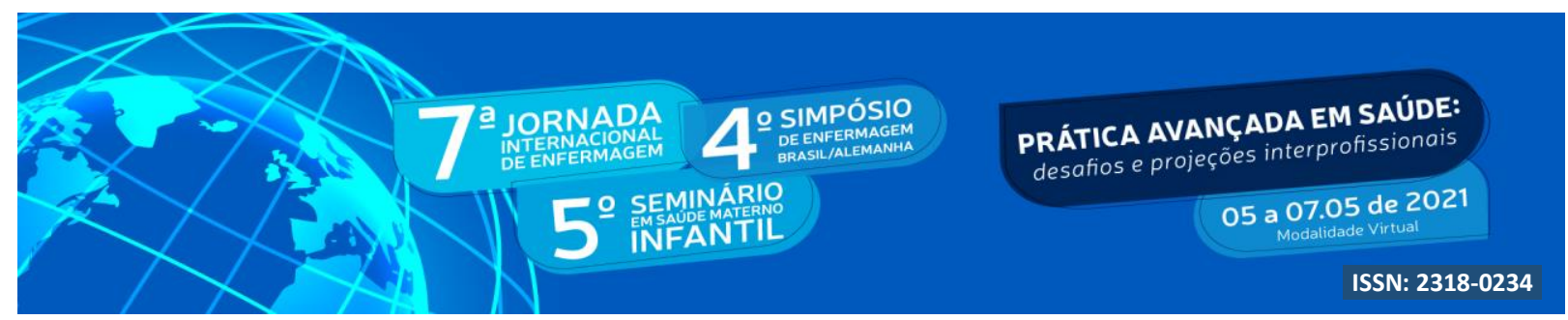

stages: Construction and validation of the pediatric first aid protocol; prototype modeling; prototyping validation; application implementation. Based on the Delphi technique, the application was considered by experts to be relevant in terms of functionality, reliability, accessibility, feasibility and innovation. The mobile first aid device for pediatric unintentional injuries will serve as a support in pediatric emergency situations for both family caregivers and health and education professionals

Key Words: First aid; Nursing care; Mobile Apps; Information Technology; Technology innovation

\section{INTRODUÇÃO}

Primeiros socorros são constituídos pela avaliação da necessidade de intervenção e consequentemente, a realização de condutas iniciais que objetivam auxiliar pessoas que estejam em sofrimento ou risco de morte, qualquer pessoa com conhecimento dos procedimentos pode realizá-los, mesmo que não seja profissional de saúde (Singletary et. al., 2015; Galindo et al., 2018). A fim de conferir acesso e segurança aos indivíduos no enfrentamento de situações adversas, a abordagem dos primeiros socorros deve se democratizada a fim de conferir acesso e segurança aos indivíduos no enfrentamento de situações adversas (Galindo et., al 2018).

As injúrias não intencionais (acidentes domésticos) representam a principal causa de morte na faixa etária entre zero e dez anos de idade no País (ROCHA et al., 2014). Neste sentido, torna-se pertinente a educação em saúde para a população a partir do desenvolvimento de tecnologias educativas, protocolos de amplo e rápido acesso (Aliveira et al., 2015; Bezerra, 2014).

A sociedade passou por diversas mudanças tecnológicas, culturais, educacionais, se adaptar a essas mudanças a fim de propiciar a população informação de qualidade no seu processo saúde-doença, sobretudo, diante de situações adversas é fundamental e a enfermagem ocupa um espaço promissor neste âmbito. Dentre as tecnologias de apoio, aplicativos de smartphones são um exemplo por conceder acesso rápido a informações de qualquer lugar em qualquer hora e amplo alcance pela população, de tecnologia (Bezerra, 2014).

Em buscas de lojas virtuais (Play Store e Apple Store) por aplicativos para dispositivos móveis relacionados aos primeiros socorros pediátricos, não foram encontradas tecnologias 


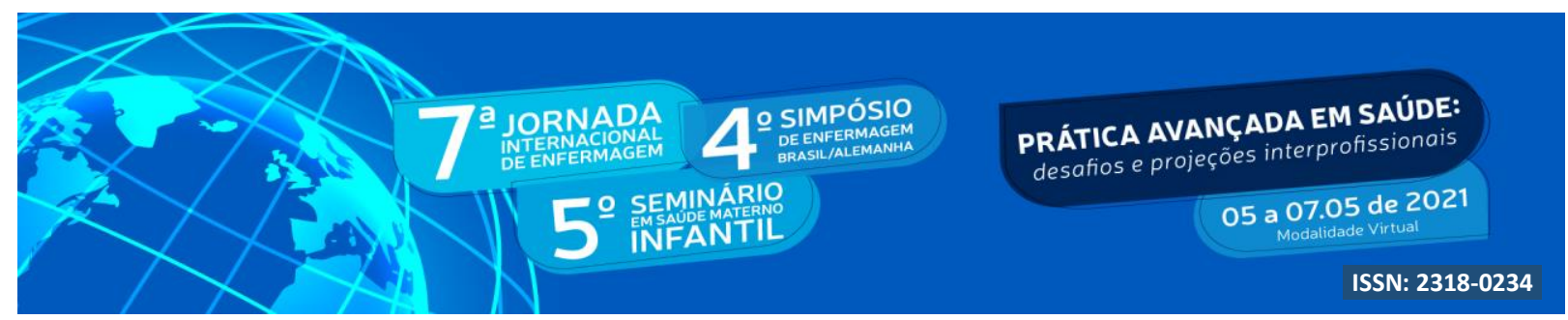

semelhantes relativas ao tópico, tanto em âmbito nacional quanto internacional. Assim, no sentido de ampliar as discussões sobre a temática, o presente estudo teve como questão de pesquisa: qual a relevância de um aplicativo para dispositivos móveis sobre primeiros socorros para injúrias não intencionais pediátricas?

\section{OBJETIVO}

Descrever o processo de desenvolvimento de um aplicativo de primeiros socorros para injúrias não intencionais pediátricas.

\section{METODOLOGIA}

Trata-se de um estudo de produção tecnológica, do tipo prototipagem, desenvolvido no Sul do Brasil, entre os anos de 2018 e 2019, a partir de quatro etapas: Construção e validação do protocolo de primeiros socorros pediátricos; modelagem do protótipo; validação da prototipagem; design centrado no usuário e implementação do aplicativo em sua forma online.

Seguindo a ordem das etapas anteriormente citadas, o processo de construção e validação do protocolo de primeiros socorros pediátricos, dividiu-se em cinco estágios: elaboração, aplicação, correção e interpretação dos resultados. Para medidas avaliativas, utilizou-se o índice de validade de conteúdo (IVC) e aparente, relacionados à contribuição de experts, na área sob investigação e segundo, à compreensão dos dados por meio da linguagem e adequação dos itens (Belucci; Matsuda 2012) .

A partir de uma revisão aprofundada, foi construído o protocolo de primeiros socorros com as principais injúrias que ocorrem em ambientes domiciliares na faixa etária pediátrica :Queimaduras, Quedas, Intoxicação/Envenenamento, Ferimentos, Afogamento, Engasgo/Sufocamento, Parada Cardiorrespiratória, Animais Peçonhentos, Choque Elétrico e Convulsão/ Epilepsia.

Em seguida foram localizados por meio da plataforma lattes e convidados, 18 profissionais, com publicações científicas na área de urgência e emergência, pediatria e neonatologia e cuidados intensivos em pacientes pediátricos. Os experts avaliaram os 


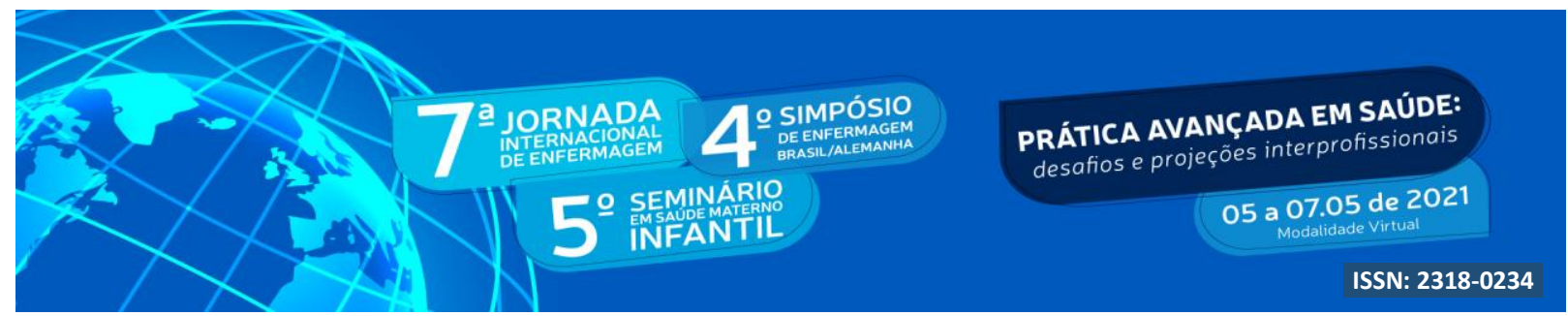

respectivos itens das cinco dimensões com base em valores numéricos, quais sejam (1) Sem importância; (2) Pouca importância; (3) Importância relativa; (4) Importante; (5) Muito importante (12). O escore do índice foi calculado por meio da soma de concordância dos itens que foram marcados por "4" ou "5" pelos experts (considerados como relevantes) divididos pelo número total de respostas (Polit, 2006; Alexandre, 2011).

Após a validação das injúrias selecionadas, construiu-se parceria acadêmica com a equipe do Laboratório de Práticas dos Cursos de Sistemas de Informação e Ciências da Computação, ambos da Universidade Franciscana (UFN) a fim de construir o modelo de protótipo do aplicativo. Nessa etapa, foram definidas as características do aplicativo e os recursos que seriam inseridos, considerando-se três critérios: 1) segurança dos dados, 2) benefícios para a população (educação em saúde acerca do tema de primeiros socorros) e 3) agilidade de acesso e clareza do conteúdo.

O protótipo foi aplicado a uma validação metodológica, efetivada com base na técnica Delphi, a partir do consenso de especialistas. Elaborou-se, para tanto, um instrumento específico de avaliação, com cinco dimensões e 18 itens. Possibilitou-se espaço, para cada item de avaliação, para que os especialistas (mencionados na primeira etapa), sugerissem modificações, caso fosse necessário, a partir de critérios já adotados em outro estudo (Turner; Zolin, 2012). A técnica de Delphi (Turner; Zolin, 2012; Belluci; Matsuda, 2012), que consiste na análise de especialistas, é realizada a partir de rodadas de avaliação de questionários com vistas ao alcance do consenso teórico do produto desejado.

A partir da validação metodológica do conteúdo informativo do software, foram desenvolvidas as telas do software. O software, possui uma tela inicial com dez ícones das injúrias não intencionais pediátricas, e um botão fixo que direciona a ligação para o SAMU (Serviço de Atendimento Móvel de Urgência e Emergência), além do ícone para informações do app e o ícone explicativo de como usá-lo. Figura 1 - Tela Inicial Aplicativo 

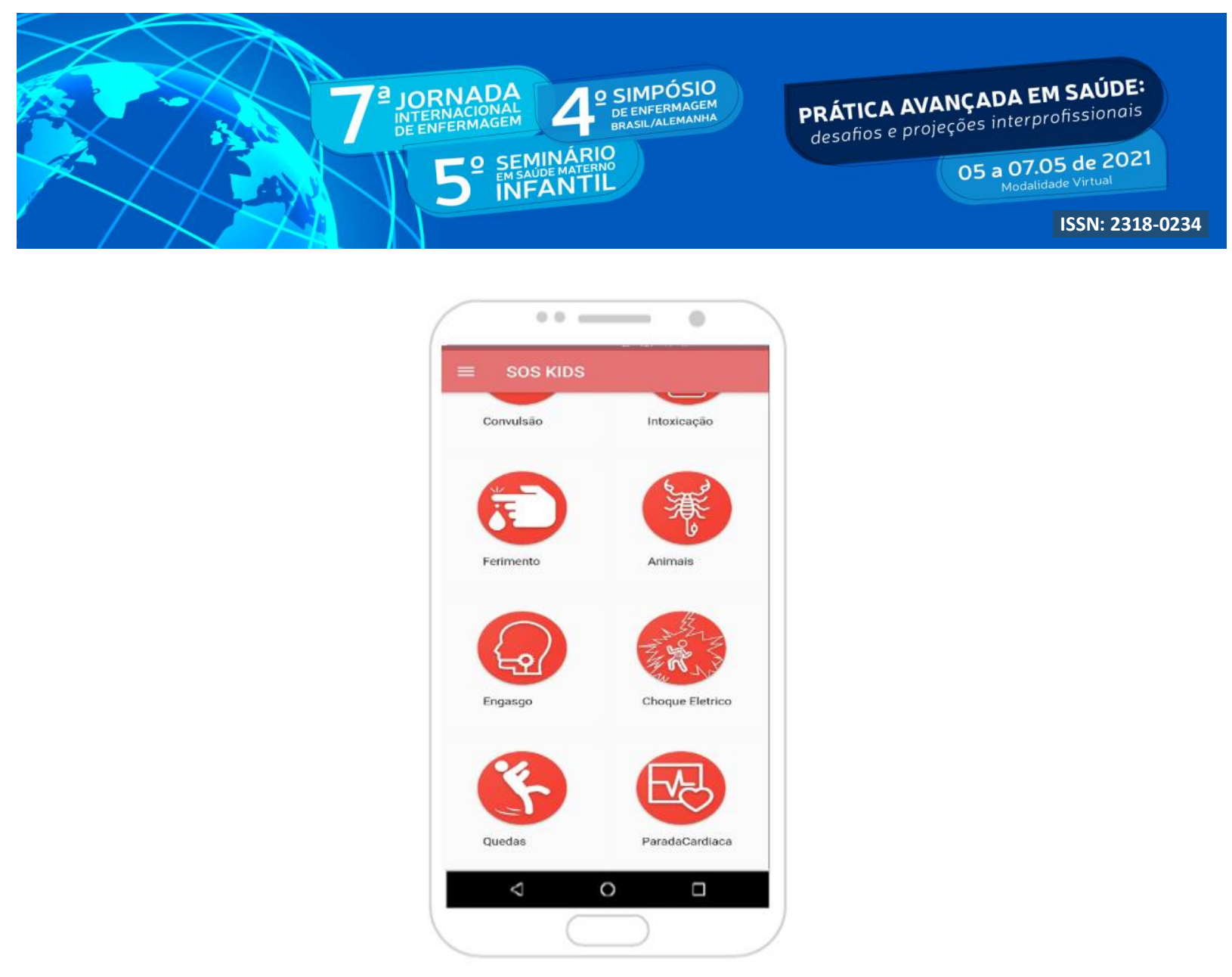

Fonte: Elaborado pelas autoras

A navegação do software é feita selecionando a injúria desejada. Após a escolha, caso a injúria tenha uma subcategoria. Após selecionar o tipo de queimadura, o navegador direciona automaticamente para a conduta a ser tomada, conforme segue:

Figura 4 - Tela Queimaduras Aplicativo 

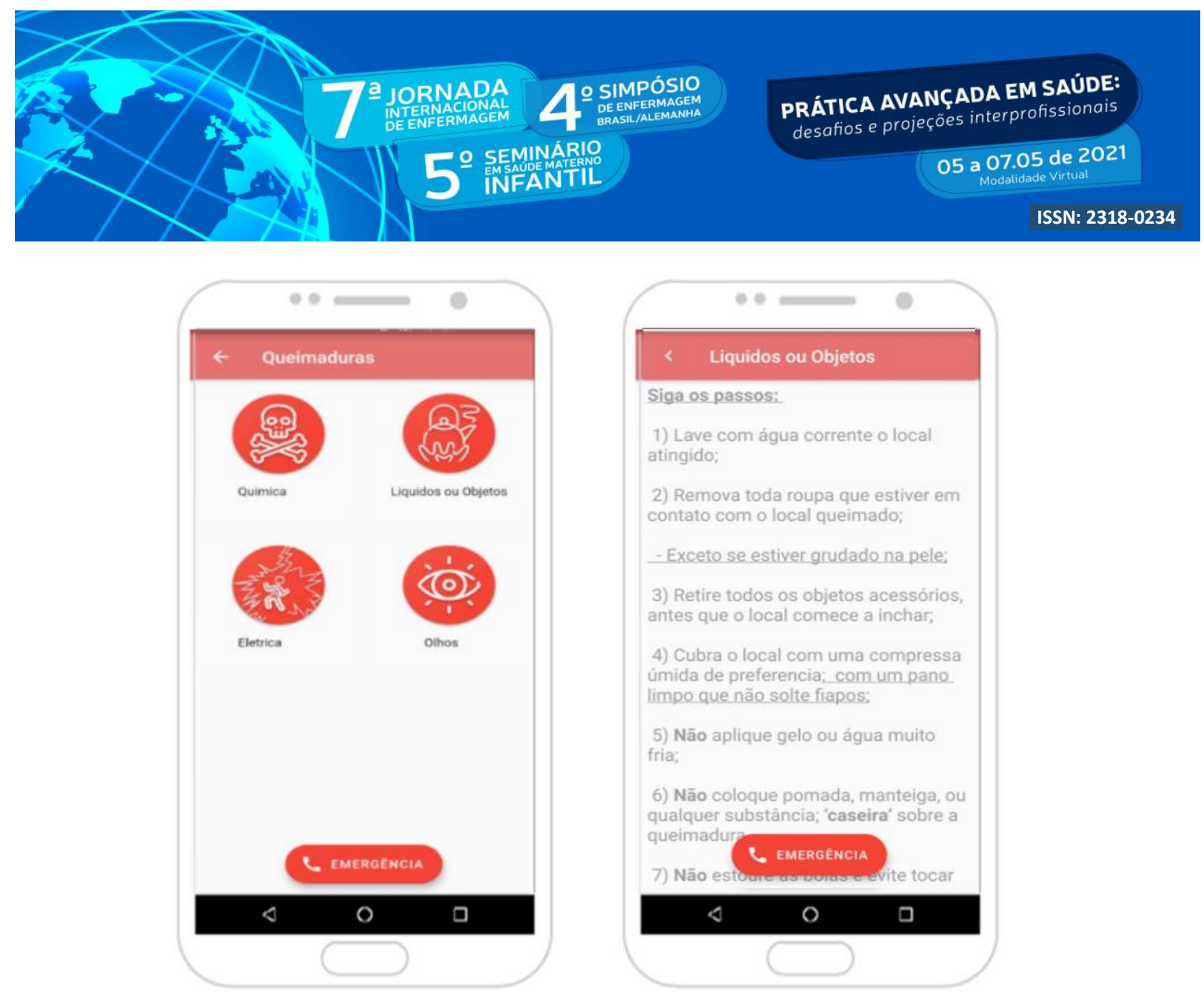

Fonte: Elaborado pelas autoras

Definido o design definitivo do protótipo de aplicativo, passou-se a realizar encontros periódicos de análise e discussão entre pesquisadores, profissional técnico e especialistas $\mathrm{O}$ projeto foi aprovado pelo Comitê de Ética em Pesquisa, sob o $n^{\circ} 2.542 .044 / 2018$, conforme Resolução 466/2012 do Conselho Nacional de Saúde.

\section{RESULTADOS E DISCUSSÃO}

O grupo de experts para a construção e a validação do protocolo de primeiros socorros pediátricos foi composto por 14 profissionais da área sob investigação, entre estes, 78,57\% são do sexo feminino com experiência em serviço variando entre 06 e 37 anos de trabalho. Em relação à titulação, $20 \%$ possuem título de especialização, $30 \%$ possuem título de mestrado, $50 \%$ possuem título de doutorado, sendo que destes, 71,42\% experts dedicam-se à área da docência. 


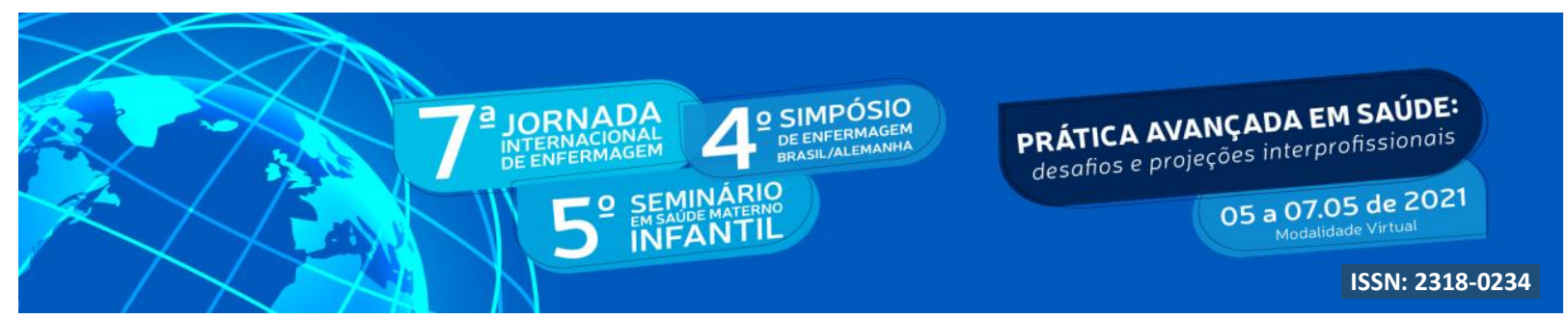

Os experts sugeriram a adoção de uma linguagem informal, a fim de facilitar a explicação nos procedimentos de primeiros socorros, como também recomendaram a utilização de materiais de fácil acesso, por se tratar de um protocolo direcionado para os cuidadores, em geral.

Em relação ao percentual de concordância dos experts, na primeira rodada, considerando a pontuação Likert entre 4 e 5, apresentou a média de 92,85\%. Já, na segunda rodada, o percentual de concordância entre os experts, considerando a pontuação Likert entre 4 e 5, foi de $100 \%$. O IVC total do instrumento foi concluído com 96,42\% de concordância entre os experts.

Em seu desenvolvimento, por meio de encontro entre os profissionais envolvidos na construção, foi priorizado um aplicativo prático e de acesso rápido, com ou sem internet, ou seja, o acesso à internet é necessário apenas para fazer o download do aplicativo. O mesmo disponibilizará as orientações em qualquer espaço, com ou sem sinal telefônico e internet.

Estabeleceu-se, para o desenvolvimento e design do aplicativo, parcerias com um profissional da área Sistemas de Informação, com a Agência Tecnológica da instituição proponente e serviços de saúde, com os quais foram realizadas reuniões periódicas para o alinhamento do processo com base nas cinco dimensões previamente validadas. Tratou-se, além disso, de características de funcionalidade específicas e do nome definitivo do aplicativo que, dentre outras sugestões.

Recentemente implementada, a lei 3.722, de 04 de outubro de 2018, enfatiza que todos os professores e funcionários de escolas deverão ter noções básicas de primeiros socorros. $\mathrm{O}$ que enfatiza a necessidade de dispositivos de atualização, como as tecnologias de fácil acesso para direcionar as condutas, visto que os professores vivenciam situações de primeiros socorros e por não praticar frequentemente tem medo e muitas vezes não lembram da conduta correta (Brasil, 2018). Corrobora a Política Nacional de Redução de Morbimortalidade por Acidentes e Violências uma vez que contribui com a multiplicação de informações aos professores, pais e cuidadores referentes às condutas corretas a serem adotadas em casos de urgência e emergência (Brasil, 2018).

\section{CONCLUSÃO}




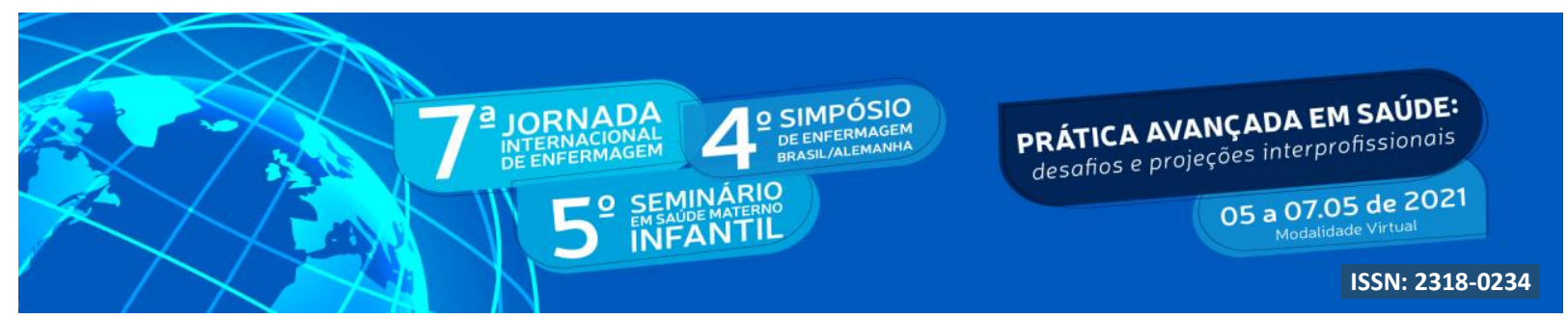

O dispositivo móvel desenvolvido é uma ferramenta que oferecerá apoio e acesso de informação de qualidade sobre primeiros socorros para injúrias não intencionais pediátricas, não apenas para indivíduos em situações de emergência pediátrica, tanto para os cuidadores familiares, quanto para os profissionais da área da saúde e da educação. Como consequência, a utilização poderá resultar na diminuição dos índices de acidentes domiciliares, podendo ser graves com cicatrizes físicas e emocionais (algumas reabilitáveis e outras não), durante seu processo de crescimento e desenvolvimento.

O aplicativo móvel "SOS KIDS" sobre primeiros socorros pediátricos desenvolvido e validado, será de relevância para pais, cuidadores, professores e população em geral. As etapas e critérios adotados e o referencial teórico utilizado para construção do aplicativo foram compatíveis com a obtenção de um aplicativo educativo viável para ser utilizado e de fácil acesso pelo público-alvo.

O aplicativo SOS Kids foi registrado no Instituto Nacional de Propriedade Industrial, em 01 de outubro de 2020, sob o Código: BR512020002188-3 esua versão final do aplicativo foi disponibilizada para download no Google Play da plataforma Android. Apesar do conteúdo do aplicativo ter sido validado é importante, sempre que necessário, atentar para as atualizações do protocolo, sobretudo, no que se refere ao conteúdo.

\section{REFERÊNCIAS}

ALEXANDRE NMC, Coluci MZO. Content validity in the development and adaptation processes of menasurement instruments. Ciênc Saúde Coletiva, 2011;16(7):3061-8.

AMERICAN HEART ASSOCIATION. First. Aid. Ciculation. 2005; 6:196-203.

\section{BELLUCI JUNIOR JA, Matsuda LM. Construção e validação de instrumento para} avaliação do acolhimento com classificação de risco. Rev Bras Enferm. 2012;65(5):751-7.

\section{BEZERRA SMMS. Health education conception that guides the educational practice of} nurse's caring. [editorial]. Rev Enferm UFPE on line. [periódico na Internet] 2014;8(9): 


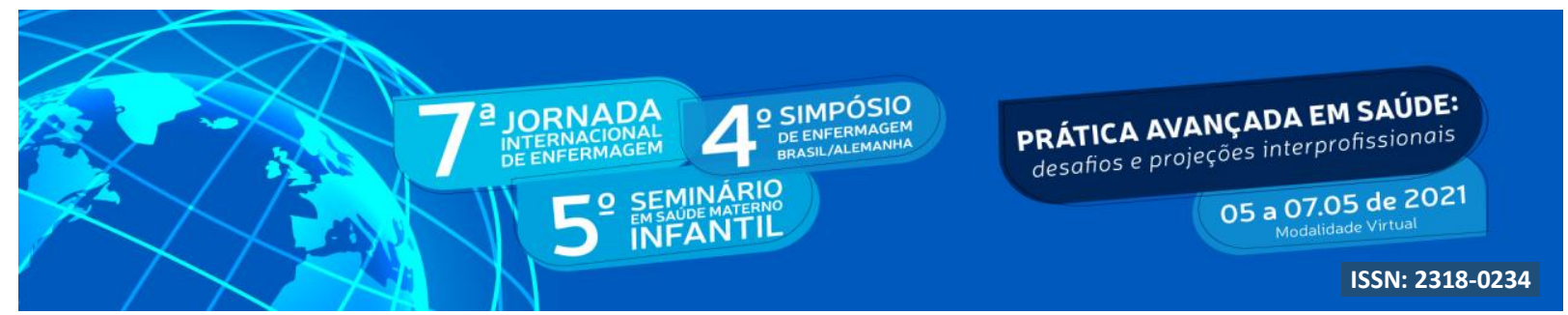

Analytics Wireless Smartphone Strategies (WSS) service, Boston, MA; 2012.

BRASIL. Ministério da Saúde. Manual de diagnóstico e tratamento de acidentes por animais peçonhentos. 2a . ed. Brasília: Fundação Nacional de Saúde; 2001.

BRASIL. Ministério da Saúde. Mortalidade. Óbitos por residência por faixa etária determinada segundo. Brasília, 2018. [acesso em 2018 jun 21] Disponível em: http://tabnet.datasus.gov.br/cgi/tabcgi.exe?sim/cnv/obtuf.def.>.

GALINDO NETO NM, Carvalho GCN, Castro RCMB, Caetano JA, Santos ECB, Silva TM, et al. Teachers' experiences about first aid at school. Rev Bras Enferm [Internet]. 2018;71(Suppl 4):1678-84. [Thematic Issue: Education and teaching in Nursing] DOI: http://dx.doi.org/10.1590/0034-7167-2017-0715

GALINDO NETO NM, Pereira JCN, Muniz MLC, Mallmann DG, Souza NMG, Neri MFS, et al. Health education intervention on first aid in school: integrative review. Int Arch Med [Internet]. 2016[cited 2017 Jan14];9(144):1-7.Available from: http://imed.pub/ojs/ index.php/iam/article/view/1733

MAGAlHÃES, Nuno Miguel Rodrigues de Sousa. A Cor como Intenção no Design de Comunicação. Tese de Doutorado, 2020.

ROCHA NETO AP, Maciel SM, Lopes MLH, Sardinha AH, Cunha CLF. Perfil dos acidentes por queimaduras em crianças menores de dez anos. Revista de Políticas Públicas. 2014 jan/jun;13(1):41-47.

SINGLETARY EM, Charlton NP, Epstein JL, Ferguson JD, Jensen JL, MacPherson AI et al. First Aid: 2015 American Heart Association and American Red Cross Guidelines Update for First Aid. Circulation. 2015;132(Suppl 2)18:574-89. 


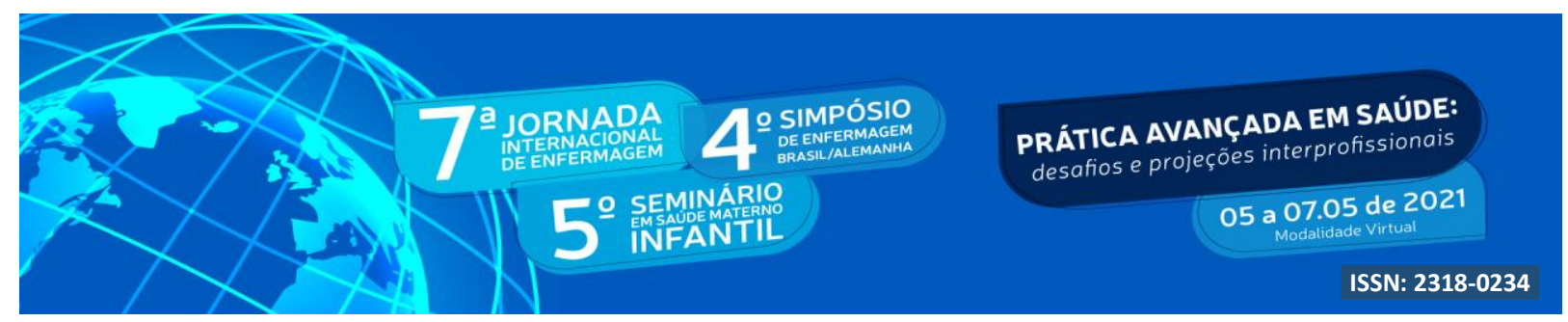

TURNER R, Zolin R. Forecasting success on large projects: developing reliable scales to predict multiple perspectives by multiple stakeholders over multiple time frames. Project Management Journal. 2012;43(5), 87-99. 